\title{
Cationic agents for DNA compaction
}

\author{
Sylwia Gawęda ${ }^{\mathrm{a}}$, M. Carmen Morán ${ }^{\mathrm{a}, *}$, Alberto A.C.C. Pais ${ }^{\mathrm{a}, *}$, Rita S. Dias ${ }^{\mathrm{b}}$, Karin Schillén ${ }^{\mathrm{b}}$, Björn Lindman $^{\mathrm{a}, \mathrm{b}}$, \\ M. Graça Miguel ${ }^{a}$ \\ a Chemistry Department, Coimbra University, 3004-535 Coimbra, Portugal \\ b Physical Chemistry 1, Lund University, P.O. Box 124, 22100 Lund, Sweden
}

\section{A R T I C L E I N F O}

\section{Article history:}

Received 15 February 2008

Accepted 4 April 2008

Available online 9 April 2008

\section{Keywords:}

DNA compaction

Coexistence

Intermediate structures

Fluorescence microscopy

Dynamic light scattering

\begin{abstract}
A B S T R A C T
Fluorescence microscopy was used to investigate the conformational changes of individual T4 DNA molecules induced by different compacting agents, namely the cationic surfactants, cetyltrimethylammonium bromide (CTAB) and chloride (CTAC), iron(III), lysozyme, and protamine sulfate. A protocol for establishing size estimates is suggested to obtain reproducible results. Observations show that in the presence of lysozyme and protamine sulfate, DNA molecules exhibit a conformational change from an elongated coil structure to compact globules, usually interpreted as a first-order transition. The maximum degree of compaction that is attained when iron(III) or CTAB (CTAC) are used as compacting agents is considerably smaller, and intermediate structures (less elongated coils) are visible even for high concentrations of these agents. Dynamic light scattering experiments were carried out, for some of the systems, to assess the reliability of size estimates from fluorescence microscopy.
\end{abstract}

(C) 2008 Elsevier Inc. All rights reserved.

\section{Introduction}

Deoxyribonucleic acid (DNA) is a nucleic acid that contains the genetic instructions for the development and functioning of living organisms. The main role of DNA in the cell is the long-term storage of information. DNA is a long anionic polymer, soluble in aqueous solutions. The ability of DNA to bind polycation molecules yielding polyplexes is widely used in biotechnological applications such as nonviral gene delivery [1], regulation of gene expression, and replication of DNA [2,3].

It was found that, in aqueous solutions, DNA chains usually exhibit an elongated coil state if no compacting agent is present [4]. However, DNA molecules exist in a highly packed state in living cells. It has been found that isolated DNA chains undergo a coilglobule transition upon the increasing concentration of condensing agents in solution [5-7]. Chemical agents cause compaction by modifying electrostatic interactions between DNA segments, by modifying DNA-solvent interactions, by excluding volume to the worm-like coil (and/or counterions), by causing localized bending or distorsion of the helical structure, or by some combination of these effects. Compaction of DNA chains is induced in vitro by the addition of agents such as polyamines [8], multivalent metal cations [9], hydrophilic polymers [10], cationic polymers [11,12], catanionic liposomes [13], and cationic [14] and nonionic surfac-

\footnotetext{
* Corresponding author.

E-mail addresses: mcarmen@qui.uc.pt (M.C. Morán), pais@qui.uc.pt (A.A.C.C. Pais).
}

tants [15]. Also, it may be induced by changes in the relative permittivity of the medium [16].

DNA condensation has been observed by a variety of techniques that detect changes in polymer size, including various forms of electron microscopy, total intensity and dynamic laser light scattering, sedimentation, viscometry, linear optical dichroism, and circular dichroism. An important addition to this list is fluorescence microscopy (FM), which was used to observe the condensation of single large T4 DNA molecules [5,6,17]. It has been used in a large number of studies on the interaction between DNA and different compacting agents $[4,9,10,14]$. Commonly, observations are consistent with a phase diagram that comprises three regions. First, in the absence, or for low concentrations of the compacting agent, all the DNA macromolecules display an extended conformation exhibiting a slow "worm-like" motion (coil). For higher concentrations of most compacting agents, only compact structures (globules) are visible [9]. Intermediate states, corresponding to intrachain segregation or extended local folding, are usually not found [18] and, for intermediate concentrations of the compaction agent, the two populations coexist in solution.

An extensive number of studies with different compacting agents has been presented, in particular by Yoshikawa and his group. Normally it is found convenient to distinguish only between extended and compacted DNA molecules and most studies have focused on monitoring the relative amounts of the two states as a function of the concentration of the compacting agent. However, recent work by Yoshikawa et al. [19] has demonstrated that the simple picture of only two states is an over-simplification and that a deeper analysis is needed. 
Furthermore, computer simulations on the effect of oppositely charged cosolutes on the conformation of a polyion have demonstrated, first, that different cosolutes produce different compacted states and, second, that the compacted state depends on the cosolute concentration [20,21].

These considerations lead us to reexamine the matter. Using very recent microscopy equipment, we can now further detail the compaction behavior. In our opinion this is important not only for a deeper understanding but also for applications, as it is expected that, for example, transfection efficiency depends on the degree of compaction. In the present study, the folding transition of T4 DNA from coil structures to globules is investigated. Several condensing agents with different binding characteristics are considered: two basic proteins, lysozyme, which has 9 positive charges per molecule, and protamine sulfate, with 21 positive charges. These two proteins have different total charge and linear charge density. Other compacting agents used in this study are the metal cation iron(III) and the surfactant cetyltrimethylammonium with two different counterions, bromide and chloride (CTAB and CTAC, respectively). The purpose of the present study is to compare the performance of different condensing agents and assess the existence of intermediate structures, multichain aggregates, and other conformational aspects. A final objective is to establish a reproducible method to estimate chain sizes resorting to fluorescence microscopy, and to assess the importance of the blurring effect.

\section{Materials and methods}

\subsection{Materials}

Bacteriophage T4 DNA (165.6 kilobase pairs, contour length of $57 \mu \mathrm{m})$ was purchased from Wako Nippon Gene. The fluorescence dye 4',6-diamidino-2-phenylindole (DAPI) (excitation/emission = $360 / 460 \mathrm{~nm}$ ), the antioxidant, 2-mercaptoehanol (ME), and the buffer salt, Trizma base were purchased from Sigma. Cetyltrimethylammonium bromide and cetyltrimethylammonium chloride (CTAB and CTAC, respectively), supplied from Sigma, were re-crystallized with acetone and ethanol. Iron(III) chloride hexahydrate was purchased from Panreac and used as received. Lysozyme from chicken egg white $(14.3 \mathrm{kDa})$ and protamine from salmon in the sulfate salt $(5.1 \mathrm{kDa})$ were purchased from Sigma and used as received. All experiments were performed using Millipore Milli-Q deionized water (18.2 $\mathrm{M} \Omega / \mathrm{cm}$ resistivity).

\subsection{Sample preparation}

Solutions were prepared as described in what follows. T4 DNA was diluted with $\mathrm{pH} 7.610 \mathrm{mM}$ Tris $\mathrm{HCl}$ buffer containing $4 \%(\mathrm{v} / \mathrm{v})$ $\mathrm{ME}$ and DAPI. The final concentrations (DNA $0.25 \mu \mathrm{M}$ in terms of nucleotide units; DAPI $0.25 \mu \mathrm{M}$ ) were kept constant throughout the fluorescence microscopy experiments. This concentration of DNA corresponds to half the concentration that has been used in some of the previous studies [11,13,16,17,22-25], which makes the visualization of individual DNA molecules easier via fluorescence microscopy in our recent equipment. We note that the number of chains simultaneously visible with such equipment largely exceeds those observed with older setups. More importantly, the use of diluted solutions promotes a better interpenetration of the nucleic acid and compacting agent solutions, thus minimizing unhomogeneity effects upon mixing. Under these conditions, the binding number of DAPI per 1 bp of DNA in aqueous buffer solutions is estimated to be equal to 0.05 , leaving both persistence length (and contour length) unaltered [26,27]. Stock solutions of cationic agents were prepared at the desired concentrations. They were then gently vortexed and left to equilibrate for several days at $25^{\circ} \mathrm{C}$. Compaction studies were initiated by addition of the cationic agents to the sample solution, gently mixed, and left to equilibrate during $15 \mathrm{~min}$ at $25^{\circ} \mathrm{C}$ before examination with the fluorescence microscope.

\subsection{Fluorescence microscopy (FM)}

Samples were illuminated with a UV-mercury lamp (100 W Ushio Olympus). The fluorescence images of single DNA molecules were observed using a Olympus BX51M microscope, equipped with a UplanFL N $100 \times / 1.30$ oil-immersed objective lens $(\infty / 0.17 / F N$ 26.5) and a filter set type U-MNU2 (360-370 nm excitation and $400 \mathrm{~nm}$ dichromatic mirror). Images were digitized on a computer through a video camera (Olympus digital camera DP70) and were analyzed with an image processor (Olympus DP Controller 2.1.1.176, Olympus DP Manager 2.1.1.158). All observations were carried out at $25^{\circ} \mathrm{C}$. Special care was taken to clean the glass microscope slides (Marienfeld) and coverslips with ethanol thoroughly before each observation to prevent DNA degradation and precipitation onto the glass surface.

\subsection{Sampling}

The method of sampling to acquire estimates of DNA size is often absent from the literature. In what follows we describe in detail our procedure, and discuss some of the problems associated with the determination of the DNA size.

In order to evaluate changes in shape and conformation of DNA molecules, representative images of all the different systems were recorded, mostly consisting of movies 5 s long (ca. 22 frames). The apparent long-axis length of the DNA molecules $L$ was defined as the longest distance in the outline of the fluorescence image of a single DNA molecule; note that, due to the blurring effect on the images, DNA chains may appear slightly longer, exceeding by ca. $0.3 \mu \mathrm{m}$ the actual size of the chains [28]. Different approaches were tested to determine the size of DNA molecules, given in what follows as the longest axis in the two-dimensional images. In the initial approach, individual pictures were used. For some movies, the still pictures were taken from the initial, middle, and final frames. This produces, especially for free DNA or low concentrations of compacting agent, a large dispersion in size. The average value of the longest axis in the visible chains within a specific area can display a variation of $1.5-2 \mu \mathrm{m}$ among the frames. The explanation for such a result is simple. When looking at frozen conformations of the chains, the observed sizes vary not only according to the actual degree of extension of the chain, but also according to the orientation of the molecule towards the observer and the amount that is hidden in nonvisible layers of the solution. The alternative is to follow the molecules along the film, and select each chain according to the maximum size it displays. Such a procedure introduces some bias in the size, favoring larger sizes in the random behavior of chains with large conformational freedom. However, it also discards artifacts resulting from the mentioned aspects of the visualization. All results presented here are based on this approach. Analyses of the images were performed using the public domain Image J 1.31v program (http://rsb.info.nih.gov/ij/).

Furthermore, it should also be stressed that extended but partially adsorbed molecules also introduce complications. First, their random behavior sometimes obscures the fact that they are adsorbed. Second, since the observation is perpendicular to the slide, small sizes are usually ascribed to these chains. Often the brightest part is that in contact with the glass, which makes it easy to incorrectly classify such adsorbed molecules as globules. As such, determinations in size were only carried out from samples where adsorption was not detected. We note that free solution molecules are easily distinguished from adsorbed molecules by the significant difference of the Brownian motion. Note, however, that some 
molecules alternate between an adsorbed state and the presence in bulk solution.

In summary, the average of values for the longer axis length at maximum extension was calculated from 50-100 molecules, and the standard deviation determined. Note that, in what concerns the latter, our values correspond to the actual dispersion in sizes, and not to the standard deviation of the mean.

\subsection{Dynamic light scattering (DLS)}

Dynamic light scattering (DLS) measurements were performed to determine the size distribution of the DNA/CTAB complexes in aqueous solution at different concentrations of CTAB. The solutions were prepared with Tris buffer, that was filtered through $0.2 \mu \mathrm{m}$ filters from Whatman to exclude dust particles. The light scattering setup used in this study was a ALV/DLS/SLS-5000F, CGF$8 \mathrm{~F}$ goniometer system from ALV GmbH, Langen, Germany, which has been described previously [29] with the difference that cisdecahydronapthalene is used instead as the refractive index matching liquid. The light source is a diode-pumped Nd:YAG solid-state Compass DPSS laser from COHERENT, Inc., Santa Clara, CA (532 nm, $400 \mathrm{~mW}$ ). Two multiple tau digital correlators (ALV-5000/E and ALV-5000/FAST Tau Extension) with a total of 320 exponentially spaced channels were utilized to produce the time correlation function of the scattered intensity (pseudo-cross or auto). The DLS measurements were performed at $25^{\circ} \mathrm{C}$ and at different scattering angles $\left(50^{\circ}-140^{\circ}\right)$.

The measured quantity in the DLS experiments is the time correlation function of the scattered intensity. A nonlinear regularized inverse Laplace transformation procedure REPES $[29,30]$ was used to analyze the experimental intensity correlation functions to obtain the distribution of relaxation times, $A(\tau)$, where $\tau$ is the relaxation time. Here, we display the relaxation time distributions as $\tau A(\tau)=\log (\tau / \mu s)$ for equal-area representation. From the first moment of the translational mode, i.e., the relaxation rate $\Gamma(=1 / \tau)$, the translational mutual diffusion coefficient of the scattering particles can be calculated at finite concentration: $D=\Gamma / q^{2}$, where $q$ is the magnitude of the scattering vector $(q=$ $4 \pi n_{0} \sin (\theta / 2) / \lambda, n_{0}$ is the refractive index of the solvent (here: water), $\theta$ is the scattering angle and $\lambda$ is the wavelength of the incident light). In this study, $D$ was obtained as the slope of the relaxation rate measured at different scattering angles versus $q^{2}$. For dilute solutions, the apparent hydrodynamic radius $\left(R_{\mathrm{H}}^{\mathrm{app}}\right)$ of the DNA/CTAB complexes can be estimated via the Stokes-Einstein relation, $D=k T /\left(6 \pi \eta_{0} R_{\mathrm{H}}^{\mathrm{app}}\right)$, with $k$ being Boltzmann's constant, $T$ the absolute temperature and $\eta_{0}$ the viscosity of the solvent (i.e., water).

\section{Results and discussion}

\subsection{Compaction studies}

It has been a general finding that, depending on the concentration of the cationic agent, individual DNA chains exhibit an all-or-none transition between the elongated and folded compact states and that there exists a rather wide region in which the two states coexist [25]. We examine here the effect of lysozyme, protamine sulfate, iron(III), and CTAB (CTAC) on the conformation of DNA using fluorescence microscopy.

Fig. 1 shows a typical fluorescence image of T4 DNA molecules in an extended coil state, observed at $\mathrm{pH} 7.6$, in $10 \mathrm{mM}$ Tris $\mathrm{HCl}$ buffer solution and in the absence of the compacting agent. During the observation, DNA molecules exhibit a slow worm-like motion.

\subsubsection{Proteins}

We first examine how the DNA solution evolves with increasing concentration of lysozyme. For concentrations up to $0.08 \mu \mathrm{M}$,

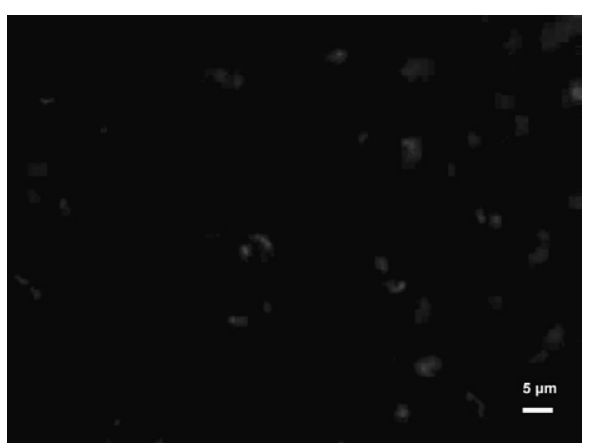

Fig. 1. Fluorescence image of unfolded T4 DNA molecules moving freely in the buffer solution at $25^{\circ} \mathrm{C}([\mathrm{DNA}]=0.25 \mu \mathrm{M})$.

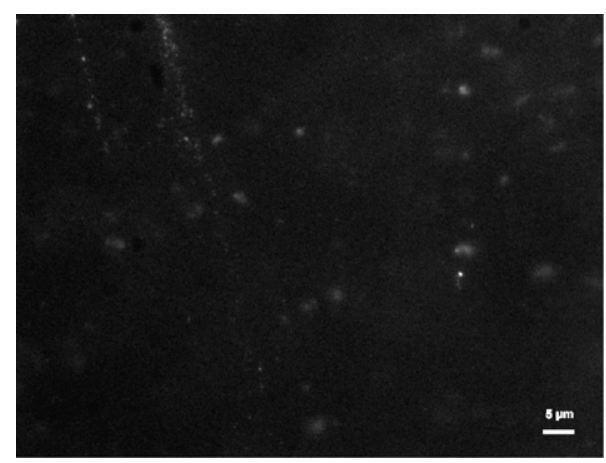

(a)

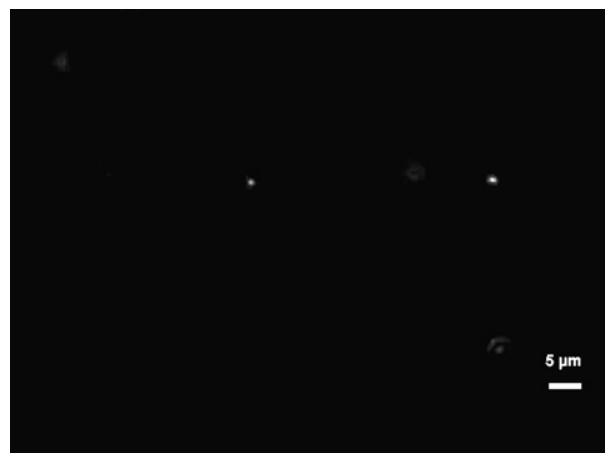

(b)

Fig. 2. Fluorescence image of T4 DNA molecules moving freely in the buffer solution at $25{ }^{\circ} \mathrm{C}$ for different concentrations of lysozyme: (a) Coexistence of coil and globule states $(3 \mu \mathrm{M})$, and (b) the existence of globule conformation alone $(5 \mu \mathrm{M})$ are shown.

only elongated coils are observed. Then, between 0.1 and $3 \mu \mathrm{M}$ there is a region in which both states, coils and globules, coexist. In the presence of $5 \mu \mathrm{M}$ lysozyme, only globules are observed. Fig. 2 shows representative fluorescence images of DNA molecules in the coiled and in the globular state.

From the fluorescence images, the distribution of the long-axis length $L$ of T4 DNA at different lysozyme concentrations was calculated (Fig. 3). The probability depicted in each histogram is normalized so that the total area is unitary. It is seen that globules start to appear at a concentration of ca. $0.5 \mu \mathrm{M}$, for $3 \mu \mathrm{M}$ they comprise about half of the chain population, and at $5 \mu \mathrm{M}$ all chains are in a compact globular state.

The conformational behavior of DNA in the presence of this protein follows the general trend described in the literature for most compacting agents. However, some points deserve being stressed. First, a higher degree of fluctuation is found for the size of the coils, compared to previous observations for most compacting agents. This has also some impact on the average values, making the overall trend (Fig. 4) slightly less defined for lower concen- 

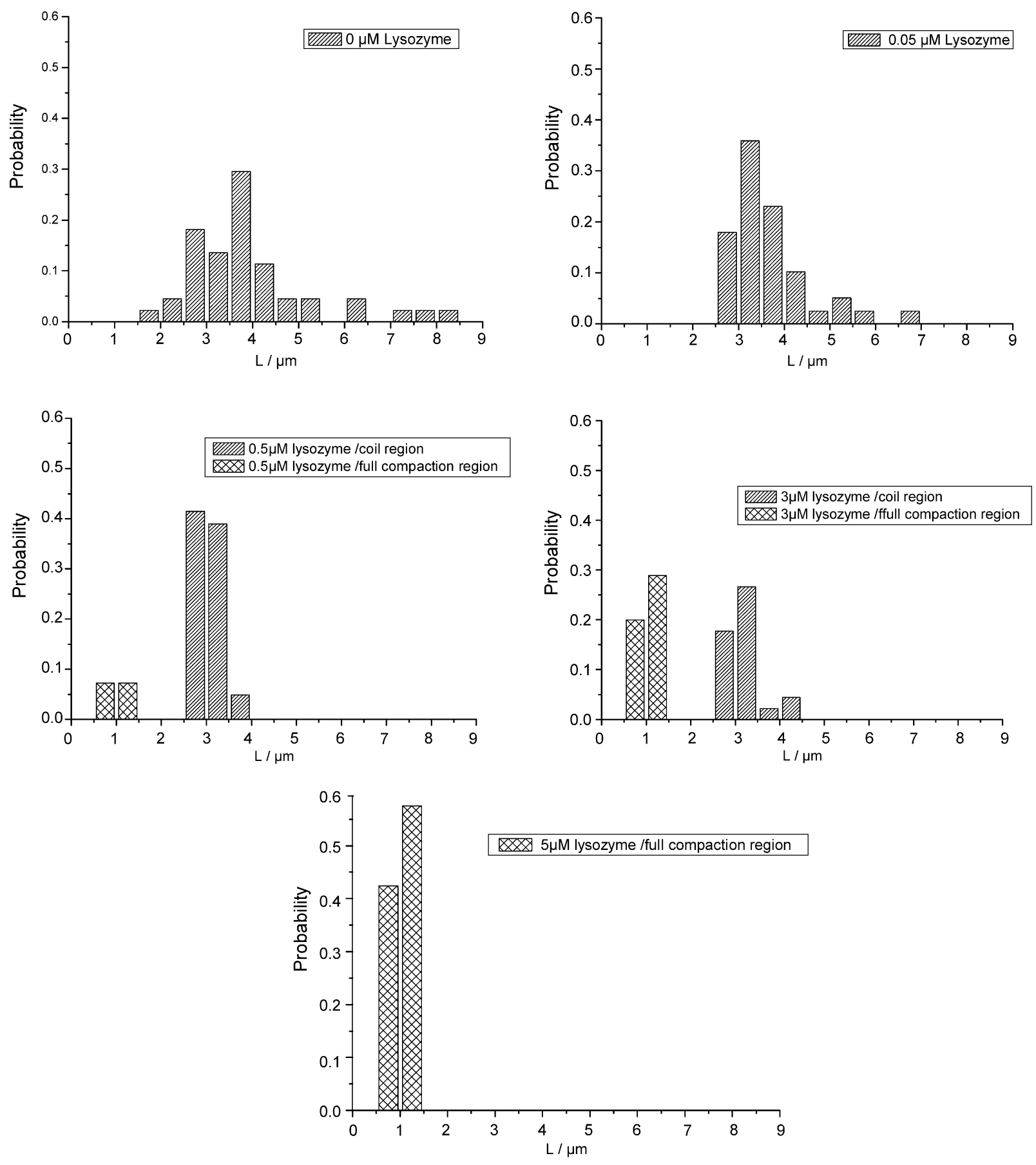

Fig. 3. Histogram of the long-axis length $L$ of T4 DNA molecules at the indicated concentrations of lysozyme. The areas in the histograms are normalized to unity.

trations of the added agent (see reference [31] in which the role of fluctuations in the degree of binding is discussed). The change in $L$ for the ensemble average (solid line) appears to be continuous, despite the all-or-none nature of the transition in individual DNA molecules, as often mentioned in previous work (see, e.g., Ref. [9]).

Also, the separation in size between coils and globules is drastic (although probably overemphasized by the accounting method). Previous experimental results have show this same separation $[9,14,22,25,32]$. Predictions on the action of polycation chains upon model DNA molecules are also clearly compatible with these results [20].

Fig. 4 shows, apart from a significant dispersion for low concentrations of the protein, an initial descending trend in size, in the unfolded region. The trend is not pronounced and is probably due to a certain degree of local folding in chains otherwise behaving closely as random coils. Again, this is compatible with the predictions from computer experiments [20]. The initial descent has not been highlighted in previous work resorting to similar agents (such as polyamines). We believe that it results from a more homogeneous distribution of the agent upon the DNA chains that directly results from the use of a diluted solution for the nucleic acid. After the coexistence regime the more compact structures dominate, but at higher concentrations of the protein, the globules display a certain expansion. This, or corresponding behaviours, is also predicted in a variety of charged systems $[20,21,33]$. Overcharged complexes, and screening of the electrostatic interactions are two causes of such a behaviour, compaction usually being seen as enthalpically driven, with entropic effects opposing the existence of compact 
structures. Variations in this behavior are sometimes observed for low charge cationic agents [34,35], but the observations are not totally consistent (see, e.g., Ref. [36]).

The effect of an increase in the overall charge and the charge density, relative to lysozyme, was tested by the use of protamine sulfate. The trend is very similar, and the final globular structure is also very dense. As could be expected, the coexistence region is shifted to lower concentrations. The first globules are visible at $0.025 \mu \mathrm{M}$, and total conversion to globules occurs at approximately $1 \mu \mathrm{M}$. Some compact structures obtained with protamine sulfate are shown in Fig. 5a. It is seen that the average size of globules, $1.14 \pm 0.17 \mu \mathrm{m}$ is similar to that of lysozyme. Fluorescence

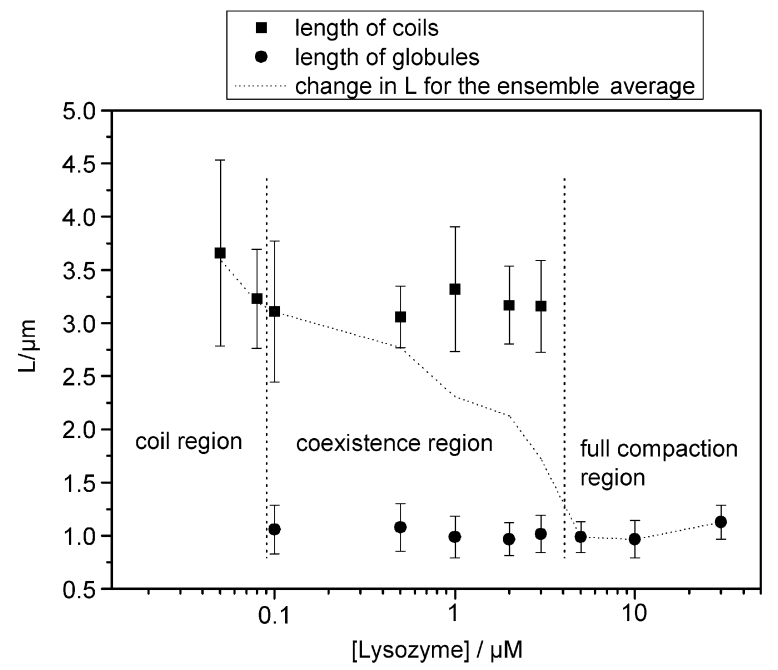

Fig. 4. Average values of the longest axis in the DNA chains observed at different lysozyme concentrations. The closed squares and closed circles indicate the values of $L$ in coiled and globular DNA molecules, respectively. Error bars display the dispersion in the distribution, and are given as one standard deviation. microscopy does not allow for an unequivocal size discrimination between the globules formed with lysozyme and protamine sulfate. Also, some degree of conformational freedom is visible in the compact structures for both agents. They maintain the reduced dimensions, but the respective shape varies with time. This is a clear indication that size estimates in terms of long-axis lengths from fluorescence microscopy are useful, i.e., although blurring effects may introduce a certain degree of uncertainty and essentially lead to an overestimation, the order of magnitude of the estimate is nevertheless correct. Most globules are, for both cases, mobile, i.e., they do not adsorb to the glass surface.

\subsubsection{Iron[III]}

We have also investigated a comparatively less charged agent, a metal ion, iron(III), for which studies have previously been reported [9]. In the presence of ascorbic acid, this agent can be easily reduced to iron (II), which would cause clear decompaction in most circumstances (relatively low concentrations and good solvent). The compact structures obtained with this agent are shown in Fig. 5b, which corresponds to a $6 \mu \mathrm{M}$ solution of the metal ion. For these conditions, the average long-axis length of the globules is $1.71 \pm 0.17 \mu \mathrm{m}$, and it is not substantially modified for higher iron concentrations. This size is significantly larger than that found for the corresponding compact structures obtained when the proteins are used. Also the internal freedom of the chains is even higher than that found for both proteins, indicating that the estimate is valid. The fact that the degree of compaction induced by trivalent metal ions is not strong has also been pointed out in simulation experiments [37]. Observations for these model systems suggest compaction arising from a closeness of relatively extended loops, formed under the local action of the trivalent ions. We note that the metal ions are not capable of promoting, e.g., bridging effects between parts of the chain far apart, contrary to what may happen with polycation chains. Thus, it is not surprising that the latter produce more compact structures.

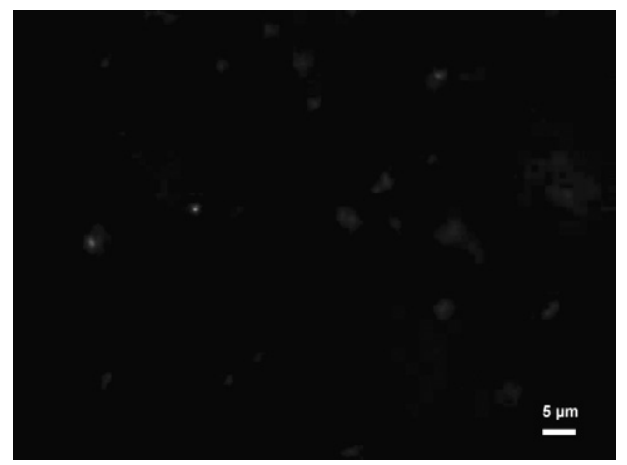

(b)

(a)
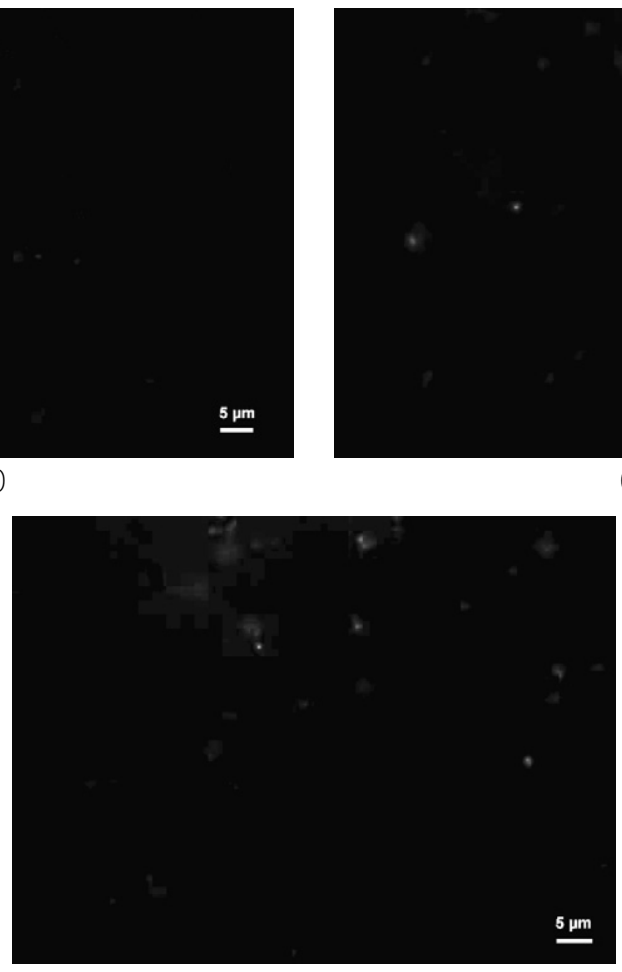

(c)

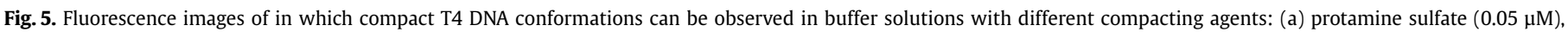
(b) iron(III) $(6 \mu \mathrm{M})$, and (c) CTAB $(40 \mu \mathrm{M})$. 

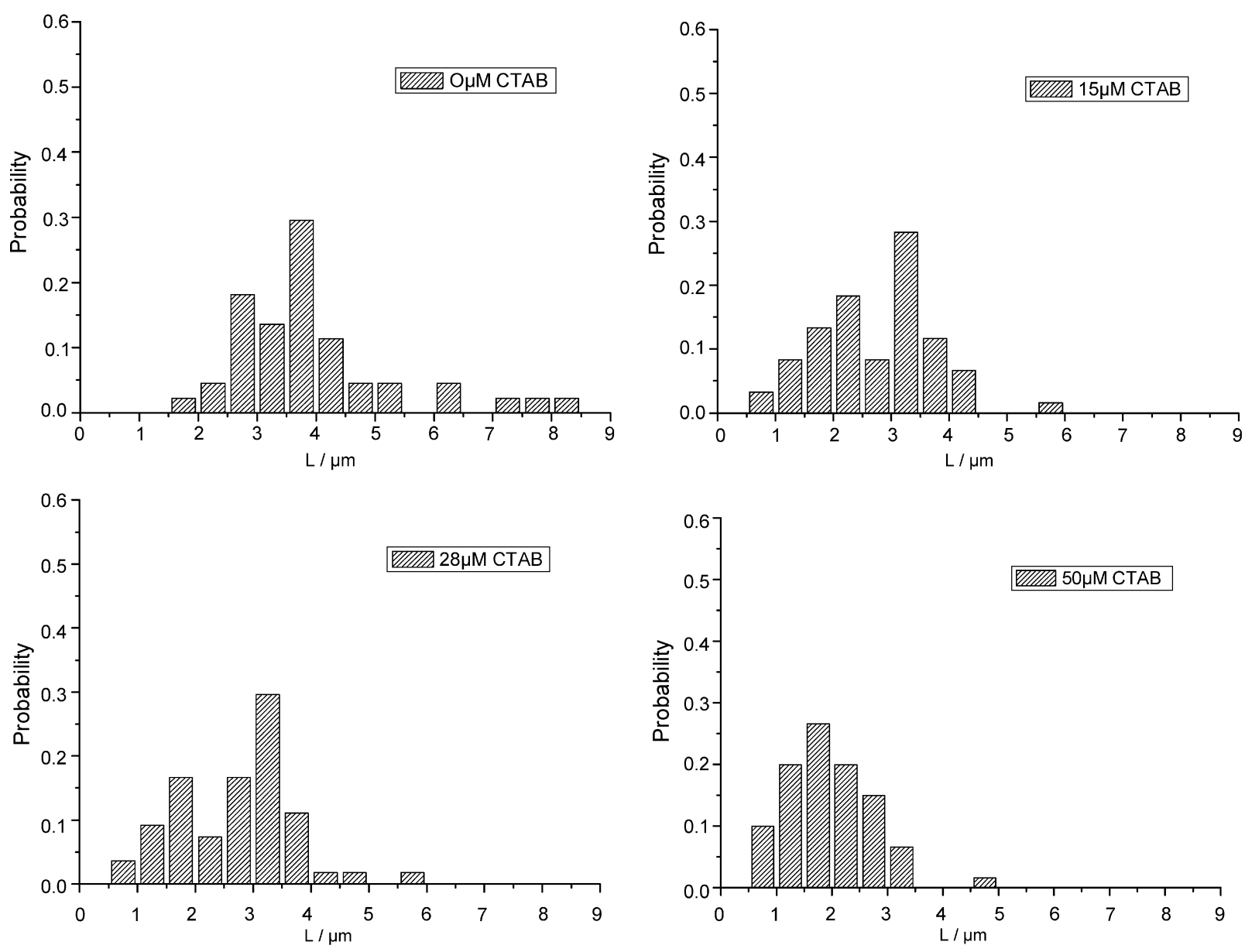

Fig. 6. Histograms of long-axis length $L$ of T4 DNA molecules at various concentrations of CTAB $([\mathrm{DNA}]=0.25 \mu \mathrm{M})$.

\subsubsection{Cationic surfactants}

Studies were also carried out using CTAB as condensing agent. This surfactant has been previously used to compact DNA [23], but here we will highlight some different aspects, found under the present experimental conditions, and monitored in new equipment.

It was observed that, as the surfactant concentration is increased, the size of the structures decrease, but the degree of compaction is always low (Fig. 5c). It is difficult to identify, in our observations, conformations that may unequivocally be classified as globules; however, for high concentrations of the compacting agent, the size of the structures is clearly reduced. Also, the fluctuations in size are always very large, which is consistent with the fact that the aggregates tend to maintain a large degree of conformational freedom. These observations are illustrated in Fig. 6, which collects size histograms for the CTAB/DNA system at selected concentrations.

Irrespective of the concentration, sizes are distributed in a continuous way. Unlike the proteins considered in this work, the surfactant departs from the 'all-or-nothing' picture usually considered for a first-order phase transition [32]. Intermediate structures are present for most concentrations. We will not attempt to establish the distinction between globules and coils. However, Fig. 7 presents some trends that are compatible with previous observations [14]. First, the overall behaviour consists of a decrease in size as the surfactant concentration increases. Also, when the concentration of СТАВ exceeds $28 \mu \mathrm{M}$, there is a steeper decrease. A plateau is observed starting at $30 \mu \mathrm{M}$.

One question that deserves further analysis is why in some previous studies [14,22], coexistence is found for CTAB/CTAC, in

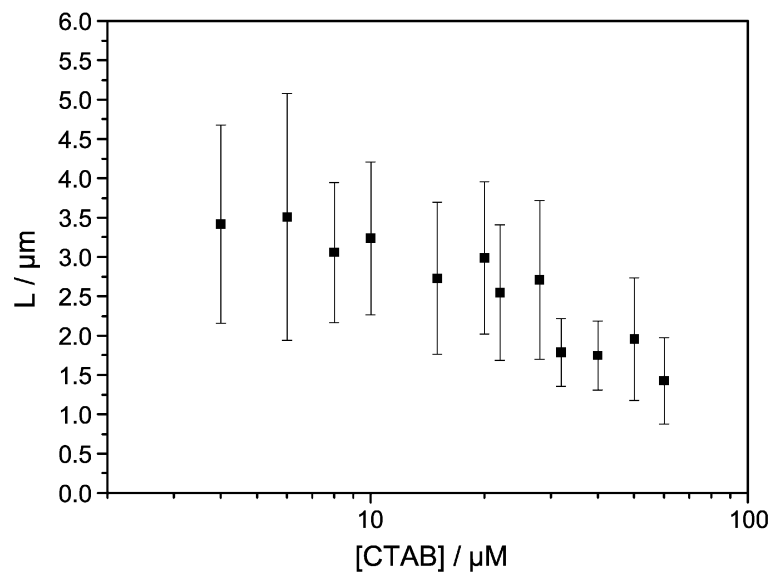

Fig. 7. Average values corresponding to the distributions in Fig. 6.

the mentioned 'all-or-nothing' scheme. A tentative explanation is a different concentration of the DNA solutions. In our case, a more diluted solution is used, which means that a more homogeneous distribution of the surfactant molecules around the DNA chains is to be expected. On the other hand, for a higher concentration of the nucleic acid, the initial fluctuation of surfactant concentration immediatelly upon mixing is likely to have more impact in the binding. We note that some of these effects are essentially irreversible (at least for the time of observation). For higher DNA concentrations, some molecules will bind much more surfactant than others, turning into globules, in the perspective of the 'doublecooperativity' mechanism. A final note to point that the behavior 


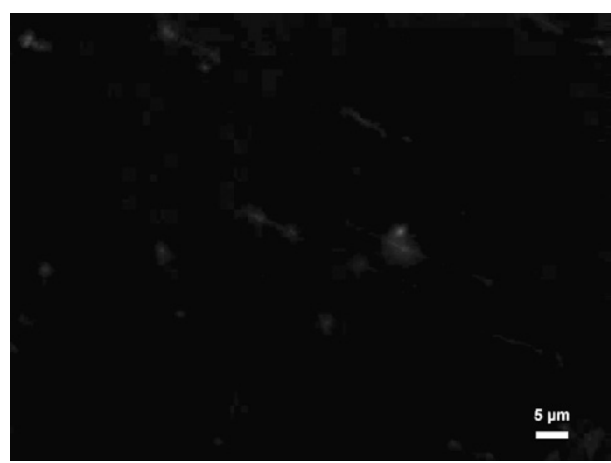

Fig. 8. Fluorescence images of T4 DNA molecules in the presence of cationic surfactant CTAB $(24 \mu \mathrm{M})$ with stretched conformations resulting from flow in the solution $([\mathrm{DNA}]=0.25 \mu \mathrm{M})$.

found for these cationic surfactants seems to be comparable to that observed for divalent cations [38].

We note that another characteristic of these systems with DNA and CTAB is the frequent adsorption to the glass. In the presence of a certain degree of flow in the solution under observation, some chains become extensively stretched, as can be seen in Fig. 8 .

It is well known that the fluorescence of many fluorophores is quenched by heavy-atom quenchers such as bromine and iodine [39]. However, chloride is a less effective quencher and relatively few fluorophores are quenched by chloride [40]. The quench- ing effect of the bromide ion in CTAB was observed in our studies (Fig. 9). As a consequence, for higher concentrations, the brighter spots (usually this part is in contact with the glass) are visible, but other parts of the chain become difficult to discern. Note in Fig. 9a how the structures can easily be mistaken by globules. CTAC behaves in a very similar fashion to CTAB, but is exempt from this quenching action. In Fig. 9b we can clearly see the tails protruding from the glass surface. Fig. $9 c$ is a schematic representation of the observed behavior.

Larger aggregates are also present in these systems (see Fig. 10), and have been previously mentioned in connection with studies of cationic gemini surfactant/DNA systems (see Fig. 1 of Ref. [24]). Our observations suggest that they are transient structures resulting from incompletely dissolved DNA, or DNA/surfactant complexes. DNA is finding its way into bulk solution, as visible in the lower part of panel B. In fact, the nucleic acid is present in a very low concentration, and it is unlikely that bundling [41], or any other form of aggregate, is present in an equilibrium state. We believe that our observations correspond essentially to single chain structures, except for the transient aggregates.

\subsection{Size estimates from dynamic light scattering}

Some of the solutions of DNA in the presence of CTAB have also been subjected to dynamic light scattering measurements. This has allowed to estimate, independently from the fluorescence

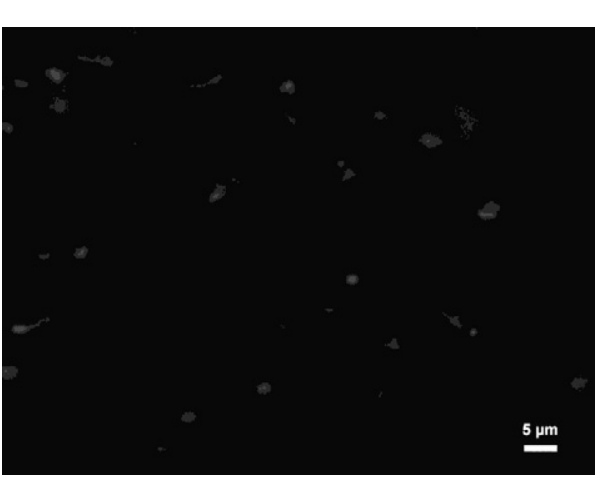

(b)

(a)

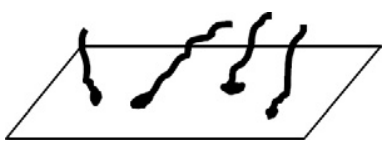

(c)

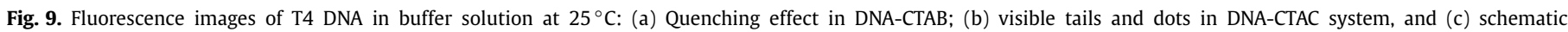
representation of the DNA-surfactant system for a high concentration of surfactant.

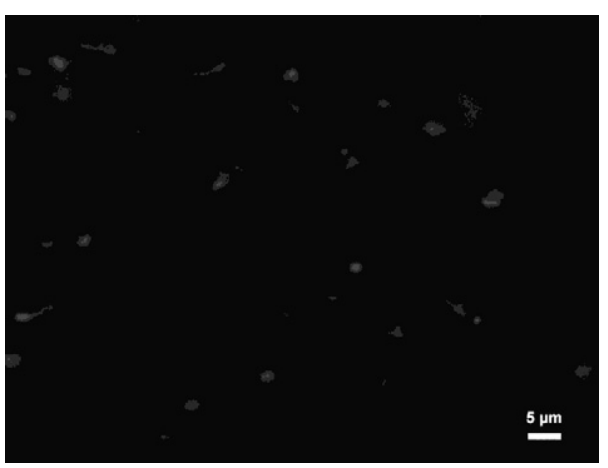

(a)

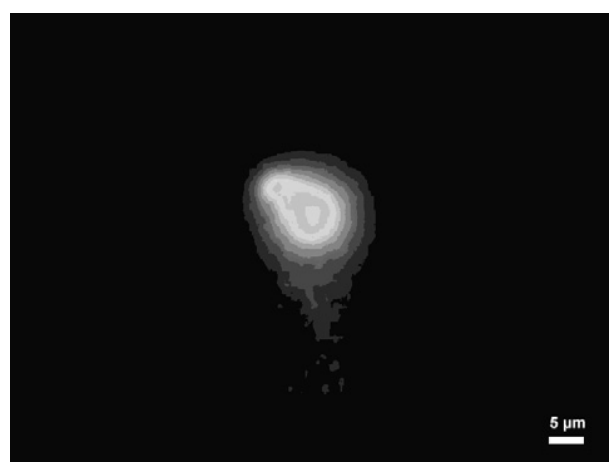

(b)

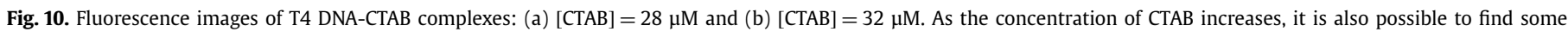
moving aggregates. 


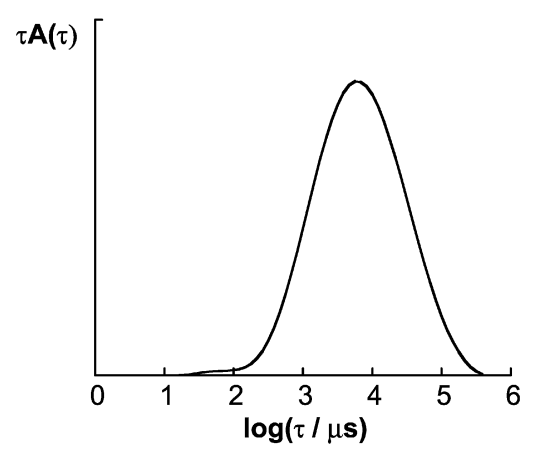

Fig. 11. A relaxation distribution obtained from the analysis of the DLS data in the globular region $([\mathrm{CTAB}] /[\mathrm{DNA}]=240)$. Measurement performed at $0.25 \mu \mathrm{M} \mathrm{DNA}$ and at $\theta=140^{\circ}$.

microscopy measurements, the hydrodynamic size of the conformational structures.

Both samples from the globular (compacted DNA state) and the co-existence regime (compacted and uncompacted state) were investigated. In these measurements, no diffusion of free DNA can be seen. Only DNA that is compacted by CTAB is observed and analyzed. Pure translational diffusion of the uncompacted DNA is only achieved at $q R_{\mathrm{g}}<1$, where $R_{\mathrm{g}}$ is the radius of gyration [42-45]. Using an estimated $R_{\mathrm{g}}$ value of about $1.5 \mu \mathrm{m}$ for the long DNA chain used in this study, all measurements were thus performed in the angular regime where $q R_{\mathrm{g}} \gg 1$. In this regime, the relaxation process from the unperturbed DNA is largely influenced by the internal relaxation of the coil. Due to this, in addition to low concentration used as well as the form factor of the large DNA coil, the scattering intensity of the unperturbed DNA chain will be too low to be observed in the correlation functions and hence the translational diffusion of the extended DNA is not observed in the DLS measurements.

In the globular regime (high CTAB concentrations) DLS measurements at different angles were performed at two different DNA concentrations, 0.25 and $0.125 \mu \mathrm{M}$ (diluted from $0.25 \mu \mathrm{M}$ ), keeping the $[\mathrm{CTAB}] /[\mathrm{DNA}]$ molar ratio of 240 constant. Fig. 11 shows a selected relaxation time distribution obtained from inverse Laplace transformation of the intensity correlation function measured at $\theta=140^{\circ}$. The single mode is related to the translational diffusion of the DNA/CTAB globules.

As noticed, distribution is rather broad, which reflects a size polydispersity of the CTAB-induced DNA globules. The apparent diffusion coefficients of the globules at both DNA concentrations were obtained from the slopes of the relaxation rates as a function of $q^{2}$ (data not shown). These, and the estimated values of the apparent hydrodynamic radius, $R_{\mathrm{H}}^{\mathrm{app}}$, are collected in Table 1 . The values are rather close to each other. The small difference reflects that interparticle interactions are present in the system. For accurate size determinations, extrapolations to infinite dilution should be made.

In the coexistence regime at intermediate CTAB concentrations, the measurements were more challenging to perform since the DNA chains in this regime are more extended and the scattering power of the solutions was therefore weaker. The DNA concentration needed to be increased to $1 \mu \mathrm{M}$ in order to be able to achieve good statistics and good correlation functions. The relaxation time distributions obtained for a CTAB concentration of $96 \mu \mathrm{M}$ were dominated by a large broad mode at longer times, which sometimes was accompanied by a faster low-amplitude mode. The large mode was attributed to the translational diffusion process of the large DNA/CTAB complexes. There was too little information in the correlation functions to further analyze the small mode and we therefore only present the data obtained from the large mode, from which a hydrodynamic size may be estimated (Table 1 ).
Table 1

Translational diffusion coefficients, $D$, and hydrodynamic radius, $R_{\mathrm{H}}^{\text {app }}$ for DNA/CTAB complexes at $T=25^{\circ} \mathrm{C}$

\begin{tabular}{llc}
\hline Sample & $D \times 10^{13}\left[\mathrm{~m}^{2} \mathrm{~s}^{-1}\right]$ & $R_{\mathrm{H}}^{\mathrm{app}}[\mathrm{nm}]$ \\
\hline $\begin{array}{l}\text { Coexistence region } \\
1 \mu \mathrm{M} \text { DNA }\end{array}$ & $2.10 \pm 0.10^{\mathrm{a}}$ & $1172 \pm 59$ \\
{$[\mathrm{CTAB}] /[\mathrm{DNA}]=96$} & $6.40 \pm 0.30$ & $379 \pm 18$ \\
Globule region & & \\
$0.125 \mu \mathrm{M}$ DNA & & $420 \pm 5$ \\
{$[\mathrm{CTAB}] /[\mathrm{DNA}]=240$} & $5.79 \pm 0.08$ & \\
Globule region & & \\
$0.25 \mu \mathrm{M}$ DNA & & \\
{$[\mathrm{CTAB}] /[\mathrm{DNA}]=240$} & & \\
\hline
\end{tabular}

a The errors in $D$ were estimated from the errors of the linear $\Gamma$ vs $q^{2}$ fits.

The data of Table 1 reveal that the DNA/CTAB complexes are larger in the region where co-existence (complexes and unperturbed DNA chains) is expected than in the region where only globules are found in the fluorescence microscopy (FM) images. The light scattering estimate of the hydrodynamic radius in the coexistence region at [CTAB]/[DNA] $=96$ is $R_{\mathrm{H}}^{\mathrm{app}} \sim 1200 \mathrm{~nm}$ and, in what concerns FM, the average value of $L$ for that concentration is $2600 \mathrm{~nm}$. This yields an average radius of ca. $1000 \mathrm{~nm}$ after blurring is discarded. The size of the more compact structures obtained in this study for that system $([\mathrm{CTAB}] /[\mathrm{DNA}]=240)$ is given as $R_{\mathrm{H}}^{\mathrm{app}}$ (the average value) $\sim 400 \mathrm{~nm}$ with light scattering, while microscopy points to $600 \mathrm{~nm}$.

Previous results of DLS [22] on a similar system have shown the presence of a more definite coexistence behavior. However, there is some difference in the experimental conditions that may explain lesser fluctuations in the present work, i.e., sizes are more homogeneous for each concentration. In general, the dimensions found are similar to those presented here, especially for the more compact structures.

\section{Summary}

In this study, we have investigated the use of several condensing agents with different binding characteristics to promote single chain compaction of DNA. We highlight the difficulties in the use of FM for studies of the compaction of DNA. A rigid protocol must be followed to unequivocally establish reliable size distributions, and artifacts must be carefully discarded before observations are accepted.

When considering large oppositely charged polyelectrolytes, such as the proteins lysozyme and protamine sulfate, it was shown that these are very efficient compaction agents. The conformational behavior of DNA in the presence of both proteins follows the general trend described for most cases: a discrete transition between expanded coil and condensed globule, with a coil-globule coexistence region. As expected, considering the number of positive charges, the amount of lysozyme required to initiate DNA compaction is larger than that of protamine sulfate and the coexistence region for the first system spans a larger interval of concentrations.

By addition of the multivalent ion, iron(III), a limited degree of compaction is induced. This fact is in agreement with the results obtained by simulation of the compaction induced by trivalent metal ions [37]. Observations for these model systems suggest compaction arising from an approach of relatively extended loops, formed under the local action of the trivalent ions. We note that the metal ions are not capable of promoting, e.g., bridging effects between parts of the chain far apart, contrary to what may happen with polycation chains. We also note that in previous work [9], the relatively low compacting efficiency of this agent was not stressed. In that work, estimates for the globule size were much lower (inferior to $1 \mu \mathrm{m}$ ). A tentative explanation for this fact is that globules 
are characterised by a very bright central part (the core that concentrates most of the dye). Thus, in a less sensitive equipment, the outer part is not visible and, thus, the size estimate is lower. In summary, these ions and polyions of different valencies clearly promote different degrees of compaction in the chains, under the described experimental conditions.

From the agents considered in this work, the cationic surfactants (CTAB and CTAC) are the least efficient. The more compact structures obtained are large, in relative terms. The evolution of the system is also significantly different with these agents. Intermediate structures, i.e., partially folded chains are visible in what corresponds to the coexistence region of the more efficient, polycationic agents. The general trend in this region is a gradual reduction of the extension of the chain, similar to what has been observed for divalent ions [46] and spermidine [47]. A clear separation between coils and globules is not possible, but the final degree of compaction is similar for iron(III) and CTAB/CTAC. Size estimates from FM and DLS are very similar for these systems.

Finally, it is interesting to note that, in spite of the fact that these type of studies resort to very low concentrations for the DNA solutions, the single chain behavior is still affected by such concentration.

\section{Acknowledgments}

This work was supported by grants from the Fundacao para a Ciencia e Tecnologia (FCT, POCTI/QUI/45344/02 and POCTI/QUI/ 58689/2004) and a grant from an EU Research Training Network, CIPSNAC (Colloidal and Interfacial Properties of Synthetic Nucleic Acid Complexes) (contract number: MRTN-CT-2003-504932).

\section{References}

[1] K. Yoshikawa, Y. Matsuzawa, K. Minagawa, M. Doi, M. Matsumoto, Biochem. Biophys. Res. Commun. 188 (1992) 1274-1279.

[2] F. Livolant, Physica A 176 (1991) 117-137.

[3] L.D. Murphy, S.B. Zimmerman, Biochim. Biophys. Acta 1219 (1994) 277-284.

[4] M. Matsumoto, T. Sakaguchi, M. Kimura, K. Doi, K. Mingawa, Y. Matsuzawa, K. Yoshikawa, J. Polym. Sci. B Polym. Phys. 30 (1992) 779-783.

[5] R.S. Dias, A.A.C.C. Pais, M.G. Miguel, B. Lindman, Colloids Surf. A Physicochem. Eng. Aspects 250 (2004) 115-131.

[6] V.A. Bloomfield, Curr. Opin. Struct. Biol. 6 (1996) 334-341.

[7] V.A. Bloomfield, Biopolymers 44 (1997) 269-282.

[8] M. Takahashi, K. Yoshikawa, V.V. Vasilevskaya, A.R. Khokhlov, J. Phys. Chem. B 101 (1997) 9396-9401.

[9] Y. Yamasaki, K. Yoshikawa, J. Am. Chem. Soc. 119 (1997) 10573-10578.

[10] K. Yoshikawa, Y. Matsuzawa, Physica D 84 (1995) 220-227.

[11] S.M. Mel'nikov, V.G. Sergeyev, Y.S. Mel'nikova, K. Yoshikawa, J. Chem. Soc. Faraday Trans. 93 (1997) 283-288.

[12] M.L. Örberg, K. Schillén, T. Nylander, Biomacromolecules 8 (2007) 1557-1563.
[13] S.M. Mel'nikov, R.S. Dias, Y.S. Mel'nikova, E.F. Marques, M.G. Miguel, B. Lindman, FEBS Lett. 453 (1999) 113-118.

[14] S.M. Mel'nikov, V.G. Sergeyev, K. Yoshikawa, J. Am. Chem. Soc. 117 (1995) 99519956.

[15] S.M. Mel'nikov, K. Yoshikawa, Biochem. Biophys. Res. Commun. 230 (1997) 514517.

[16] S.M. Mel'nikov, M.O. Khan, B. Lindman, B. Jönsson, J. Am. Chem. Soc. 121 (1999) $1130-1136$.

[17] S.M. Mel'nikov, V.G. Sergeyev, K. Yoshikawa, Visualization of DNA-surfactant interactions with fluorescence microscope, in: S.G. Pandalai (Ed.), Recent Research Developments in Chemical Sciences, Trivandrum, India, 1997, pp. 69113.

[18] Y. Yamasaki, Y. Teramoto, K. Yoshikawa, Biophys. J. 80 (2001) 2823-2832.

[19] A.A. Zinchenko, T. Sakaue, S. Araki, K. Yoshikawa, D. Baigl, J. Phys. Chem. B 111 (2007) 3019-3031.

[20] R.S. Dias, A.A.C.C. Pais, M.G. Miguel, B. Lindman, J. Chem. Phys. 119 (2003) 8150-8157.

[21] J.M.G. Sarraguça, M. Skepö, A.A.C.C. Pais, P. Linse, J. Chem. Phys. 119 (2003) 12621-12628.

[22] R.S. Dias, J. Innerlohinger, O. Glatter, M.G. Miguel, B. Lindman, J. Phys. Chem. B 109 (2005) 10458-10463.

[23] R.S. Dias, M. Rosa, A.A.C.C. Pais, M.G. Miguel, B. Lindman, J. Chin. Chem. Soc. 51 (2004) 447-469.

[24] L. Karlsson, M.C.P. van Eijk, O. Söderman, J. Colloid Interface Sci. 252 (2002) 290-296.

[25] K. Yoshikawa, Y. Yoshikawa, T. Kanbe, Chem. Phys. Lett. 354 (2002) 354-359.

[26] Y. Matsuzawa, K. Minagawa, K. Yoshikawa, M. Matsumoto, M. Doi, Nucleic Acids Symp. Ser. 25 (1991) 131-132.

[27] Y. Matsuzawa, K. Yoshikawa, Nucleos. Nucleot. Nucl. 13 (1994) 1415-1423.

[28] K. Yoshikawa, Adv. Drug Delivery Rev. 52 (2001) 2235-2244.

[29] J. Jansson, K. Schillén, G. Olofsson, R.C. da Silva, W. Loh, J. Phys. Chem. B 108 (2004) 82-92.

[30] P. Stepanek, in: W. Brown (Ed.), Dynamic Light Scattering: The Method and Some Applications, Oxford Univ. Press, Oxford, UK, 1993, p. 177.

[31] J.M.G. Sarraguça, R.S. Dias, A.A.C.C. Pais, J. Biol. Phys. 32 (2006) 421-434.

[32] K. Yoshikawa, M. Takahashi, V.V. Vasilevskaya, A.R. Khokhlov, Phys. Rev. Lett. 76 (1996) 3029-3031.

[33] D.G. Angelescu, P. Linse, Langmuir 19 (2003) 9661-9668.

[34] D. Matulis, I. Rouzina, V.A. Bloomfield, J. Mol. Biol. 296 (2000) 1053-1063.

[35] D. Matulis, I. Rouzina, V.A. Bloomfield, J. Am. Chem. Soc. 124 (2002) 7331-7342.

[36] R.A. Hutchins, J.M. Crenshaw, D.E. Graves, W.A. Denny, Biochemistry 42 (2003) 13754-13761.

[37] M.O. Khan, B. Jönsson, Biopolymers 49 (1999) 121-125.

[38] A.A. Zinchenko, V.G. Sergeyev, K. Yamabe, S. Murata, K. Yoshikawa, Chem. Bio. Chem. 5 (2004) 360-368.

[39] J.R. Lakowicz, Principles of Fluorescence Spectroscopy, second ed., Plenum, New York, 1999.

[40] C.D. Geddes, K. Apperson, J. Karolin, D.J.S. Birch, Anal. Biochem. 293 (2001) 6066.

[41] T. Iwataki, S. Kidoaki, T. Sakaue, K. Yoshikawa, S.S. Abramchuk, J. Chem. Phys. 120 (2004) 4004-4011.

[42] D.E. Smith, T.T. Perkins, S. Chu, Macromolecules 29 (1996) 1372-1373.

[43] K. Wakabayashi, N. Sasaki, K. Hikichi, J. Appl. Polym. Sci. 76 (2000) 1351-1358.

[44] S. Yi, K.-S. Seo, Y.-H. Cho, Sens. Actuators A 120 (2005) 429-436.

[45] Y.C. Chan, Y. Zohar, Y.-K. Lee, MEMS (2006) 438-441.

[46] A.A. Zinchenko, V.G. Sergeyev, K. Yamabe, S. Murata, K. Yoshikawa, Chem. Bio. Chem. 5 (2004) 360-368.

[47] S. Takagi, K. Tsumoto, K. Yoshikawa, J. Chem. Phys. 114 (2001) 6942-6949. 